\title{
A utilização de Softwares e Plataformas Online no Ensino da Matemática
}

\author{
Thais A. Souza , Carla L. Rodriguez \\ Centro de Matemática, Computação e Cognição, Universidade Federal do ABC Av. \\ dos Estados, 5001 - Bairro S. Terezinha, Santo André, SP, Brasil - CEP: 09210-580 \\ thais.amorimealuno.ufabc.edu.br, c.rodriguez@ufabc.edu.br
}

\begin{abstract}
This article presents the results of a Systematic Mapping of the literature on the use of online software and platforms in the teaching-learning process of Mathematics. The mapping pointed out that there are educational resources that are approached, but in a less comprehensive, productive and inclusive way. Finally, the study provided elements for planning a face-to-face workshop on function related to the use of Geogebra, which was adapted to occur synchronously online due to the outbreak caused by the new coronavirus (COVID-19).
\end{abstract}

Resumo. Este artigo apresenta os resultados de um Mapeamento Sistemático da literatura sobre a utilização de softwares e plataformas online no processo de ensino-aprendizagem de Matemática. O mapeamento apontou que existem recursos educativos que são abordados, porém de uma forma pouco abrangente, produtiva e inclusiva. Por fim, o estudo forneceu elementos para o planejamento de uma oficina presencial sobre função afim com o uso do Geogebra, a qual sofreu adaptações para ocorrer de forma síncrona online devido ao surto causado pelo novo coronavírus (COVID-19).

\section{Introdução}

As Tecnologias Digitais de Informação e Comunicação (TDIC) continuam avançando nos diversos setores da sociedade. Em se tratando de conteúdos online tem-se cada vez mais sites, plataformas virtuais, textos e mídias digitais, além de diversos softwares, que podem ser acessados offline. Muitos destes recursos têm o propósito de atender às demandas de aprendizagem da população em geral. Para [Costa et al. 2015] as TDIC's são ferramentas mediadoras da aprendizagem, que auxiliam no conhecimento e no processo de execução das atividades realizadas pelos alunos, essas também dão acesso à cultura tecno-popular, apesar desse aspecto ser pouco explorado nas escolas.

$\mathrm{Na}$ Matemática não é diferente, pois surgem cada vez mais ferramentas computacionais que buscam atender à demanda de conhecimentos matemáticos. Muitas destas ferramentas têm o intuito de apoiar o ensino e complementar o processo de aprendizagem, buscando agregar informação com interatividade e recursos audiovisuais em qualquer tempo e lugar. Pesquisas na área de tecnologias e educação têm sido desenvolvidas com o propósito de entender, por exemplo, como o m-learning, que é definido como a utilização de dispositivos móveis e portáteis para facilitar o acesso à informação em programas de ensino, pode contribuir no processo de ensino e aprendizagem da Matemática [Batista et al. 2010]. Outros estudos sobre possíveis modelos de 
implementação destes recursos no ambiente escolar, nos diferentes níveis de escolaridade, estão sendo realizados, pois é desejável que os alunos desenvolvam um ciclo de aprendizagem completo, e que estes recursos sejam inseridos na educação formal por meio de modelos pedagógicos [Behar et al. 2007, Batista et al.2011].

Levando esse contexto de ensino e uso dos recursos digitais gratuitos para o ambiente das escolas públicas brasileiras, faz-se notório que este ambiente em si, por atender majoritariamente jovens e crianças de classes populares, desempenha um papel importante na quebra de barreiras sociais, históricas e culturais, às quais são geradas pelas desigualdades econômicas e sociais. Pesquisas na área têm defendido que o uso pedagógico de tecnologias livres neste cenário, de forma a promover também a criatividade e a colaboração dos envolvidos, tem um grande potencial de transformação de pensamento e rompimento das barreiras impostas ao longo do tempo nas diversas camadas sociais [Wanderley,2017].

Com esse entendimento sobre o tema, este trabalho tem como principal objetivo analisar como o uso de softwares livres como o "Geogebra", o qual pode ser usado tanto em computadores como em smartphones no modo online ou offline, influencia o processo de ensino-aprendizagem da Matemática. Para tal foi realizado um Mapeamento Sistemático da Literatura (MSL), que direcionou a elaboração de uma oficina presencial sobre o conteúdo função afim, a qual foi adaptada para ocorrer online, de maneira síncrona.

\section{Proposta e Metodologia}

Nesta seção apresentamos o MSL realizado com o objetivo de analisar de forma abrangente se o uso de softwares gratuitos como o "Geogebra", e o uso de plataformas online de ensino como a "Khan Academy", ou até mesmo o uso de sites como o "Wolfram Alpha" e o "Symbolab", auxiliam no processo de ensino e aprendizagem da Matemática. Os resultados do MSL formam a base para o planejamento da oficina sobre função afim.

\subsection{Mapeamento Sistemático da Literatura(MSL)}

Um MSL é definido como uma revisão abrangente de estudos primários existentes em um tópico de pesquisa específico, que busca assim mapear evidências presentes neste tópico [Falbo, 2018]. Este método é essencial para se obter uma fundamentação teórica completa em áreas de estudo que têm muitos trabalhos relacionados, e que podem servir de base para outra ação dentro de uma pesquisa proposta. O MSL difere da Revisão Sistemática de Literatura, a qual é um método que traz uma revisão e extração profunda do tópico de interesse para a obtenção de resultados específicos e detalhados (Kitchenham, 2004). O MSL proposto neste trabalho é uma adaptação do modelo de [Petersen et al, 2008], o qual inclui a definição das questões de pesquisa, elaboração de uma string de busca, condução da pesquisa de estudos primários em bases previamente definidas, seleção de artigos por meio da análise detalhada aplicando critérios de inclusão e exclusão, classificação dos artigos pelo resumo e palavras-chaves e, por fim, a extração dos dados e a apresentação dos estudos selecionados.

\subsubsection{Definição das questões de pesquisa:}

As seguintes questões de pesquisa foram elaboradas com o intuito de conduzir a 
elaboração e execução do MSL:

- QP01 - Quais são os tipos de pesquisas desenvolvidas sobre o questionamento da Informática na Educação Matemática (IEM)? Justificativa: entender quais são os tópicos da IEM abordados nas pesquisas da área.

- QP02 - Que problemas têm sido levantados nas pesquisas sobre a inserção dessas tecnologias no ensino e aprendizagem da Matemática? Justificativa: compreender as dificuldades da inserção de tecnologias educacionais como uma forma de auxílio ao processo de ensino e aprendizagem da matemática.

- QP03- Quais as tecnologias adotadas como auxílio ao ensino da matemática ou estão

passando por processos de testes para medição da efetividade destas? Justificativa: identificar quais tecnologias educacionais estão em uso no cenário do processo de ensino da matemática ou estão passando por processos de testes empíricos.

- QP04 - Quais as conclusões sobre o uso de tecnologias no processo de ensino e aprendizagem da matemática? Justificativa: identificar quais os resultados e conclusões obtidos pela comunidade acadêmica referente ao uso de tecnologias como forma de auxílio no processo de ensino e aprendizagem da Matemática.

\subsubsection{Elaboração e execução do MSL:}

Após a elaboração das questões de pesquisa, o passo seguinte estava direcionado à elaboração de uma string de busca em inglês e português para a busca automatizada nas bases digitais e bibliotecas virtuais. A primeira parte da string elaborada é composta por um termo geral utilizado em pesquisas acadêmicas ou outras referências para falar sobre o tema, são eles: "Informatics in Education" e "Information technologies (ITs) in education". A segunda parte é um aprofundamento do tema, dando ênfase para o processo de ensino e aprendizado com tecnologias, utilizando os seguintes termos: "Learning with technologies", "Facilitating Learning with Technology" e "Teaching and Learning with Technology". A terceira parte é um afunilamento da cadeia de pesquisa, pois esse trabalho aborda o uso de tecnologias no ensino da Matemática, nesta seção foram utilizados os seguintes termos:"Mathematics Teaching", "Mathematics Teaching and Learning", "Mathematics education" $e$ "Teaching Mathematics". As partes quatro e cinco da string trazem algumas tecnologias que foram alvo de interesse maior deste trabalho, e os termos utilizados foram: "Online learning platforms", "eLearning Platforms", "Online Educational Platforms", "Educational software", "Educational Software Programs" $e$ "Software for Education". Posteriormente, foram definidos critérios de inclusão e exclusão para serem aplicados a todos os artigos retornados da string de busca, apresentados na Tabela 1. A ultima referência deste trabalho tem o link para acessar as referências de todos os trabalhos lidos no MSL.

Tabela 1 - Critérios de inclusão e exclusão

\begin{tabular}{|c|c|}
\hline Critérios de exclusão & Critérios de inclusão \\
\hline $\begin{array}{c}\text { Trabalhos cujo o idioma não esteja em inglês ou } \\
\text { português. }\end{array}$ & $\begin{array}{c}\text { Estudos que abordam a Informática na } \\
\text { educação devem estar limitados ao cenário } \\
\text { do processo de ensino e aprendizagem da } \\
\text { Matemática. }\end{array}$ \\
\hline
\end{tabular}




\begin{tabular}{|c|c|}
\hline Trabalhos fora do contexto de Educação \\
Matemática. & $\begin{array}{c}\text { Nos estudos empíricos é necessário um } \\
\text { detalhamento do recorte do contexto no qual } \\
\text { foi aplicado o teste e uma explicação da } \\
\text { técnica utilizada para o levantamento das } \\
\text { conclusões referentes à experiência relatada. }\end{array}$ \\
\hline $\begin{array}{c}\text { Trabalhos empíricos cujo os tópicos matemáticos } \\
\text { abordados não estejam na grade curricular do } \\
\text { Ensino Fundamental ou Ensino Médio. }\end{array}$ & $\begin{array}{c}\text { O estudo deve explorar um software ou } \\
\text { plataforma gratuita para o ensino de } \\
\text { matemática. }\end{array}$ \\
\hline $\begin{array}{c}\text { Estudos empíricos relacionados ao uso de } \\
\text { softwares, plataformas ou sites não-gratuitos. }\end{array}$ & - \\
\hline $\begin{array}{c}\text { Trabalhos empíricos com ferramentas digitais } \\
\text { educacionais que são protótipos e não estão } \\
\text { abertas ao público geral. }\end{array}$ & - \\
\hline $\begin{array}{c}\text { Trabalhos cuja leitura do texto completo é } \\
\text { realizado somente mediante pagamento. }\end{array}$ & - \\
\hline
\end{tabular}

\section{Resultados e discussão dos resultados}

Nesta seção são apresentados os resultados alcançados com o MSL, a discussão destes resultados em relação às questões de pesquisa investigadas e, por fim, os direcionamentos que apoiaram o planejamento da oficina presencial e online.

\subsection{Resultados do MSL}

QP01 - Quais são os tipos de pesquisas desenvolvidas sobre o questionamento da Informática na Educação Matemática (IEM)?

De acordo com o MSL realizado neste trabalho, dentro da IEM os tópicos mais explorados pelas pesquisas sobre a IEM estão relacionados ao Processo de Ensino e Aprendizagem (29,5\%), Tecnologias Digitais da Informação e Comunicação (TDIC) $(24,2 \%)$ e Recursos Educacionais Digitais (RED)(23,7\%). Seguidos por trabalhos que abordam a Formação de professores (10,3\%) e Informática educativa (4,2\%). O Gráfico 1 apresenta todos os tipos de pesquisa encontrados nos trabalhos selecionados.

Em relação aos métodos de pesquisa, vale destacar que diferentes métodos foram aplicados pelos estudos mapeados, por exemplo: trabalho experimental (pesquisa qualitativa), trabalho teórico (pesquisa bibliográfica) e trabalho teórico e de observação (pesquisa bibliográfica e de estudo de caso).

No que diz respeito aos assuntos matemáticos trabalhados, surgiram: geometria análitica, conceito de volume, função afim, adição de frações, conceitos geométricos, prova na matemática, frações, números híbridos, sistema de numeração decimal, multiplicação de matrizes, densidade do conjunto dos números racionais e do conjunto dos números irracionais, construção de sólidos, isometria, função quadrática, teorema de Tales, geometria, sistemas lineares, números inteiros, funções elementares, geometria hiperbólica, geometria plana, teorema de Pitágoras, teoremas de Regiomontanus, álgebra, aritmética, trigonometria, curvas cônicas, equações, função modular, congruência de 
triângulos, funções polinomiais de grau maior que dois, relações métricas e medidas de tendência central.

\section{GRÁFICO 1 - TÓPICOS DE INFORMÁTICA NA EDUCAÇÃO MATEMÁTICA (IEM)}

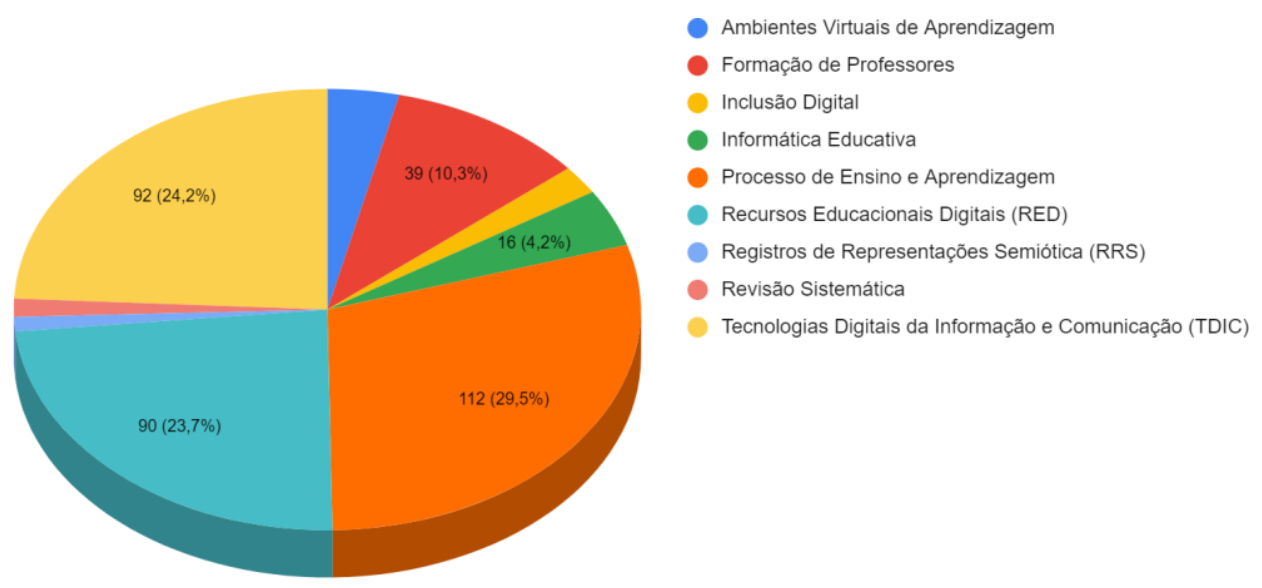

fonte: produzido pelas autoras.

QP02 - Que problemas têm sido levantados nas pesquisas sobre a inserção dessas tecnologias no ensino e aprendizagem da Matemática?

Para [Sette, 2013] a não sondagem inicial dos alunos para entender suas dúvidas e questionamentos, e a não organização temporal são fatores que atrapalham $o$ desenvolvimento de atividades com inclusão de tecnologias. [Menegais,2015] lista vários problemas, tais como: a não completa integração das TDICs como recurso de ensino e aprendizagem de Matemática, a falta de infraestrutura das escolas, conexão de internet ruim, e a não existência de conexão com a internet, além da falta de computadores e laptops. [Oliveira, 2014] também afirma que a estrutura das escolas participantes do Acessa Escola não é adequada à proposta. Em seu levantamento, o autor mostrou que os professores que diziam trabalhar com TDICs apenas as usava como inserção e não como integração. O mesmo estudo aponta que a maioria dos docentes enfrentam jornadas duplas ou até mesmo tripla, devido às suas questões financeiras, e com essas rotinas de trabalho exaustivas e falta de valorização profissional, os levam a adotar uma postura de passivos na educação.

QP03- Quais as tecnologias adotadas como auxílio ao ensino da matemática ou estão passando por processos de testes para medição da efetividade destas?

O Gráfico 2 apresenta os recursos educativos mencionados nos trabalhos estudados, os quais foram classificados em categorias genéricas. $\mathrm{OX}(21,1 \%)$ significa que no trabalho nenhum software, plataforma ou site foi abordado. É válido ressaltar que o Geogebra foi o software mais usado $(34,3 \%)$ e foi mapeado separadamente dos demais softwares. Entretanto, há estudos que utilizaram o Geogebra associado à outros recursos. 


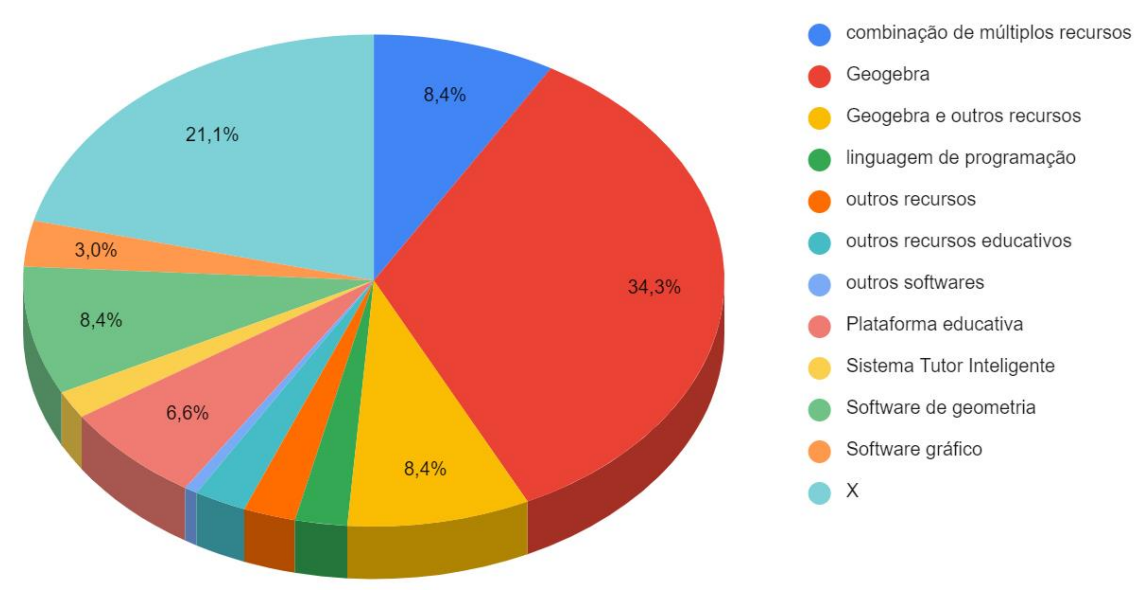

fonte: produzido pelas autoras.

QP04 - Quais as conclusões que têm sido feitas sobre o uso de tecnologias no processo de ensino e aprendizagem da matemática?

Segundo [Oliveira, 2018], as TDIC's em seu experimento contribuíram para a fluidez e a flexibilidade do pensar matemático. Para [Sette, 2013] o uso das tecnologias no desenvolvimento de atividades dá ao usuário a possibilidade de investigar, testar e levantar hipóteses. [Menegais, 2015] diz ser necessário que os cursos de formação continuada oferecidos envolvam práticas inovadoras que agreguem o contexto da sala de aula, aos quais os professores estão inseridos, e levem em consideração a individualidade de cada um. O autor também ressalta que a motivação dos professores em participar do experimento com TDIC's levou-os a um maior interesse e autonomia dos alunos. Outros estudos apontam que o profissional de Tecnologia da Informação (TI) deve atuar como coadjuvante na elaboração e avaliação de materiais didáticos, buscando agregar conceitos de interdisciplinaridade e abrindo espaço para ambientes colaborativos [Soares, 2008].

[Peres, 2015] afirma que se trabalharmos a matemática apenas de forma mecânica, não haverá uma contribuição significativa no desenvolvimento de novas habilidades pelos alunos, por isso os recursos tecnológicos são tão essenciais no âmbito escolar. Para [Rodrigues, 2011] a resolução de situações-problema com o auxílio de softwares como o Graphmatica pode beneficiar tanto o processo de ensino, quanto o processo de aprendizagem, pois desta forma o erro é visto como algo que faz parte do processo, ou seja, há uma abordagem investigativa realizada pelos alunos.

\subsection{Planejamento da Oficina Presencial e Online}

O MSL é abrangente, ainda que seja usada uma cadeia de string com afunilamento. Então, considerando os resultados obtidos foi planejada uma oficina que aborda um cenário específico para aprofundar a análise.

Para a realização da oficina presencial, as fichas de coletas de dados, o pré-teste e pós-teste, o TCLE e a proposta de intervenção seriam entregues de forma impressa aos participantes e recolhidas ao fim de cada etapa. A oficina estava prevista para ocorrer em uma escola pública, com alunos do $1^{\circ}$ ano do Ensino Médio. Porém devido ao surto do novo Coronavírus (COVID-19) no início do ano de 2020, fez-se necessário que a oficina 
passasse por um replanejamento para ocorrer de forma online. Para a adaptação ao novo formato, detalhes sobre interatividade com os participantes, conexão estável, limitação dos participantes para garantir a qualidade das informações obtidas, foram observados.

Em relação à oficina online, foram previstos três encontros virtuais síncronos: i) no primeiro encontro, a ideia é se apresentar, explicar o objetivo da oficina, conhecer os participantes e explicar brevemente sobre o Geogebra, desde a instalação até os comandos básicos; ii) no segundo encontro a ideia é realizar a oficina online com no máximo 5 participantes por sessão, sendo um total de 4 sessões. A limitação de participantes parte do princípio que a oficina seja interativa, ou seja, que os participantes compartilhem suas telas para mostrarem o que fizeram, que participem de um quiz e esclareçam suas dúvidas; iii) no terceiro encontro o objetivo é o levantamento de feedbacks e a aplicação de um pós-teste em forma de quiz.

Como materiais de apoio para condução da oficina e coleta de dados são propostos dois questionários online, um para alunos e um para professores, três aulas no formato de slides, uma para cada encontro. As oficinas estão previstas para ocorrerem de forma síncrona por meio do aplicativo Zoom, considerando a possibilidade de gravar o evento pensando nos participantes que tiverem algum imprevisto e não consigam participar. Os quizzes foram elaborados usando a aplicação Kahoot e o Google Forms. Para que os participantes não se percam caso tenham problemas com a conexão ou outros empecilhos, haverá uma pasta compartilhada com todo o conteúdo da oficina (aulas e links dos quizzes e questionários). A Tabela 2 apresenta uma comparação entre os instrumentos elaborados para as oficinas presencial e online.

Tabela 2 - Comparativo entre os instrumentos da oficina online e presencial

\begin{tabular}{|c|c|}
\hline Oficina Presencial & Oficina Online \\
\hline $\begin{array}{c}\text { Fichas coleta de dados 1 e 2 - alunos } \\
\text { A. Questões de Identificação e de Perfil - que } \\
\text { engloba identificação por nome, idade e } \\
\text { gênero. }\end{array}$ & $\begin{array}{c}\text { Questionário online - alunos } \\
\text { que engloba identificação por nome, e-mail } \\
\text { (opcional), idade, gênero, raça/cor, grau } \\
\text { máximo de escolaridade e instituição de } \\
\text { ensino. }\end{array}$ \\
$\begin{array}{c}\text { B. Questões Principais - sobre a usabilidade } \\
\text { de recursos digitais educacionais, desempenho } \\
\text { antes e após a intervenção e relato da } \\
\text { experiência com o uso de algum software ou } \\
\text { plataforma online educativa que abordam } \\
\text { assuntos matemáticos. }\end{array}$ & $\begin{array}{c}\text { B. Questões Principais - sobre a usabilidade } \\
\text { de recursos digitais educacionais, qual a } \\
\text { frequência de uso pelos estudantes, } \\
\text { eficiência de divulgação destes e quais são } \\
\text { os mais conhecidos. }\end{array}$ \\
$\begin{array}{c}\text { C. Questões Secundárias - sobre o } \\
\text { Geogebra, aprofundando aspectos da } \\
\text { experiência do usuário no processo de } \\
\text { aprendizagem. }\end{array}$ \\
$\begin{array}{c}\text { Fichas coleta de dados 1 e 2 - professores } \\
\text { A. Questões de Identificação e de Perfil - que } \\
\text { engloba identificação por nome, idade e } \\
\text { gênero. }\end{array}$ & $\begin{array}{c}\text { Questionário online - professores } \\
\text { Questões de Identificação e de Perfil - } \\
\text { que engloba identificação por nome, e- } \\
\text { mail(opcional), idade, gênero, raça/cor, grau } \\
\text { máximo de escolaridade e instituição de } \\
\text { ensino que leciona e os graus de }\end{array}$ \\
& escolaridade em que leciona. \\
\hline
\end{tabular}




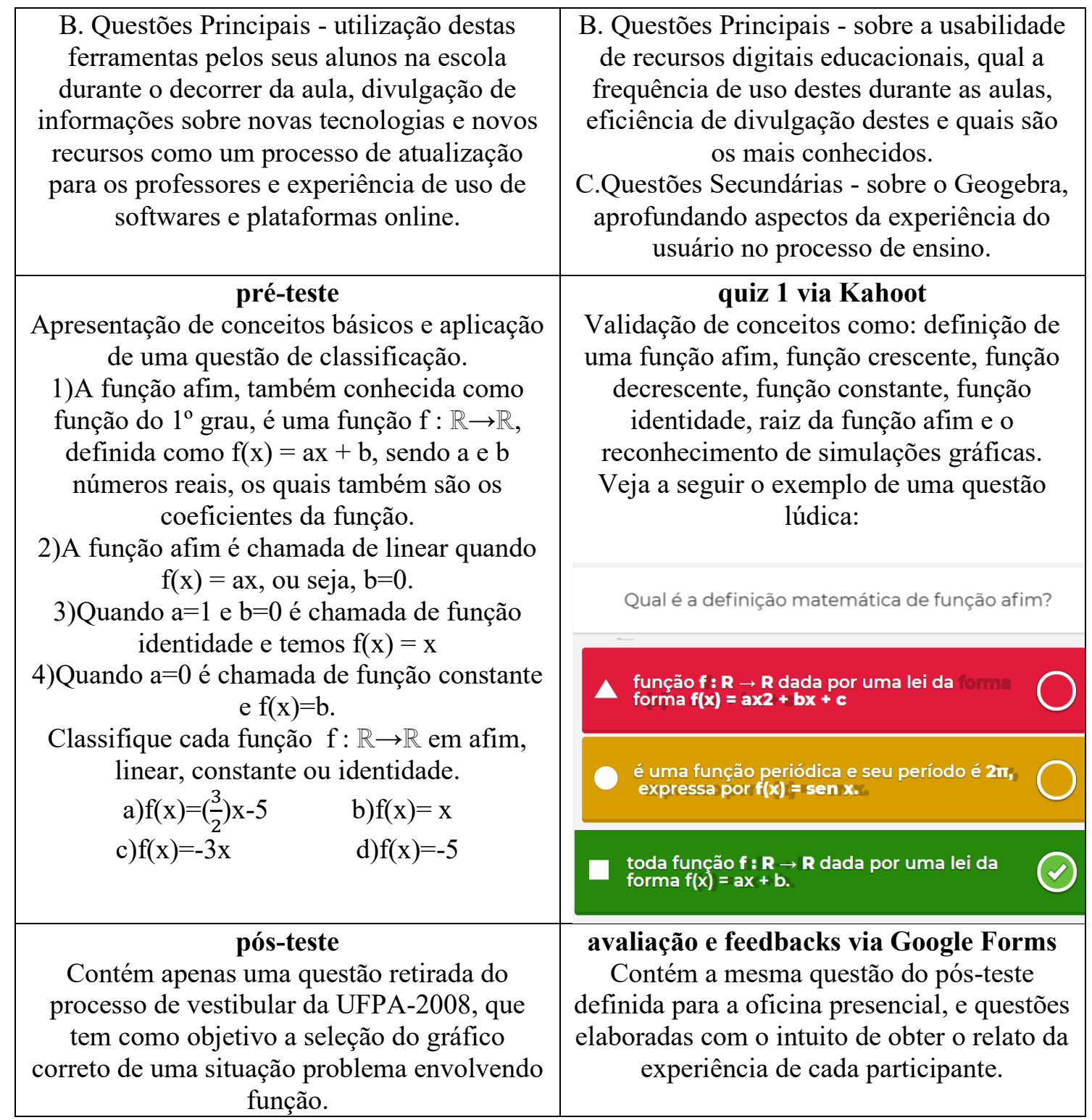

\section{Considerações Finais}

O objetivo principal deste trabalho foi analisar a utilização de softwares e plataformas online no ensino da Matemática. Para tal foi realizado um MSL cujos resultados apontam que a inclusão de TDIC's no processo de ensino-aprendizagem é positiva e benéfica desde que haja uma organização e planejamento das atividades. A Engenharia Didática é uma grande aliada neste processo. Segundo Almouloud e Coutinho (2008), as pesquisas didáticas em matemática, que recorrem à análise da experimentação, geralmente propõem uma intervenção com uma organização sistemática das observações. Outros aliados deste processo são: o Design Thinking e o Design Participativo.

A elaboração das atividades que envolvem Educação Matemática e TDIC's é um processo complexo e com duração média de tempo para sua realização. Uma maneira de tornar este processo ágil é por meio da revisão e adaptação de atividades já existentes em bancos de dados, como é o caso do portal do Geogebra que contém mais de 1 milhão de 
atividades gratuitas, simulações, exercícios, aulas e até jogos, de uso aberto [SILVA et al, 2018].

Além do planejamento e organização das atividades, é muito importante observar a questão da infraestrutura para que estas ocorram. Isto inclui ambiente adequado, conexão com a internet estável, laptops e computadores disponíveis para todos, contribuindo assim para o desenvolvimento do pensar matemático com fluidez, e permitindo que os alunos explorem outras formas de aprendizagem. De acordo com OLIVEIRA [2014], as condições de trabalho dos professores, em sua grande maioria, são precárias. Nesse sentido, além da falta de incentivo, mesmo os que tiveram acesso a cursos e oficinas de capacitação continuada não conseguem aplicar seus métodos e aprendizados em contexto de sala de aula, o que torna todo o seu conhecimento obsoleto.

Contribuindo para as reflexões na área, o relato do planejamento da oficina e sua adaptação para o ensino-aprendizagem remota mostra como a atuação do docente muda de um cenário presencial para um cenário online e síncrono. Nesse novo contexto é necessário não só se preocupar com questões de infraestrutura, planejamento da atividade, escopo e tempo, é preciso lidar com problemas de conexão, engajamento dos participantes, orientar a aprendizagem de forma remota, controle de acesso, como divulgar os encontros e materiais, como medir de forma efetiva a participação e evolução dos participantes, entre outros.

Como propostas de trabalho futuro, têm-se: (i) a realização da oficina online (realizada enquanto este trabalho estava sob revisão), com o intuito de validar o que foi levantado com o MSL; (ii) realizar um estudo de caso com os professores sobre propostas e execução de atividades que incluam o uso de softwares e plataformas online e ocorram remotamente; e (iii) desenvolver uma plataforma colaborativa na qual os educadores possam compartilhar suas atividades, incluindo estratégias de uso de tecnologias digitais.

\section{Agradecimentos}

Os autores agradecem à Pró-Reitoria de Pesquisa da Universidade Federal do $\mathrm{ABC}$ e ao CNPQ pela concessão da bolsa de Iniciação Científica e por todo o apoio para a realização deste trabalho.

\section{Referências}

Almouloud, S.A; Coutinho, S.C.Q.(2008) Engenharia Didática: características e seus usos em trabalhos apresentados no GT-19 / ANPEd ,p. 62-77. Disponível em: https://doi.org/10.5007/1981-1322.2008v3n1p62.

Batista, S.C.F; Behar P.; Passerino L. M.(2011) M-learnMat: Aplicação de um Modelo Pedagógico para Atividades de M-learning em Matemática. IN XXII Simpósio Brasileiro de Informática na Educação(SBIE). Disponível em: https://www.brie.org/pub/index.php/sbie/article/view/1810.

Batista, S.C.F; Behar P.; Passerino L. M.(2010) Recursos Pedagógicos Para Dispositivos Móveis: Uma análise com foco na Matemática. Disponível em: https://doi.org/10.22456/1679-1916.18092.

Costa, S.R.S; Duqueviz, B.C; Pedroza, R.L.S.(2015) Tecnologias Digitais como 
instrumentos mediadores da aprendizagem dos nativos digitais. Maringá ,p.603-610. Disponível em: https://doi.org/10.1590/2175-3539/2015/0193912.

Falbo, R de A.(2018) Mapeamento Sistemático. Disponível em: http://inf.ufes.br/ falbo/files/MP/TP/Sobre_MS.pdf.

DESIGN THINKING PARA EDUCADORES. Instituto Educadigital. Disponível em: https://issuu.com/dtparaeducadores.

Kitchenham, B. (2004) Procedures for performing systematic reviews. Technical Report Technical Report TR/SE-0401, Keele University and NICTA.

Menegais, D.A.F.N.(2015) A formação continuada de professores de matemática : uma inserção tecnológica da plataforma khan academy na prática docente.Disponível em: http://hdl.handle.net/10183/122036.

Oliveira, A.(2018) A Arte de Ensinar Matemática com Uso das Tecnologias Digitais. Anais do Workshop de Informática na Escola, p. 700 ISSN 2316-6541. Disponível em: http://dx.doi.org/10.5753/cbie.wie.2018.700.

Oliveira, F.T. (2014) A inviabilidade do uso das Tecnologias da Informação e Comunicação no contexto escolar: o que contam os professores de matemática? Disponível em: http://hdl.handle.net/11449/127664.

Peres, E.M.K.(2015) Apropriação de tecnologias digitais : formação continuada com professores de matemática.Disponível em: http://hdl.handle.net/10183/118896.

Petersen, K., Feldt, R., Mujtaba, S., e Mattsson, M. (2008). Systematic Mapping Studies in Software Engineering. In 12th International Conference on Evaluation and Assessment in Software Engineering (EASE), Italy, 10p.

Rodrigues, R.E de J.S.(2011) As contribuições do software Graphmatica na construção do conhecimento matemático de função. http://hdl.handle.net/11449/90987.

Sena, D. M; Oliveira, E. H. T; Carvalho, L.S.G.(2014)Aplicativos móveis para o aprendizado de matemática. IN XXV Simpósio Brasileiro de Informática na Educação.

SETTE, P. F. (2013)A aula de matemática no Projeto UCA : o Geogebra e o Teorema de Pitágoras.Disponível em: http://www.repositorio.ufop.br/handle/123456789/3475.

SILVA, R.S. R et al.(2018): Dossiê Temático: Produtos Educacionais e Educação Matemática Sobre o processo de elaboração de atividades matemáticas com o GeoGebra 3D. Disponível em: https://doi.org/10.5965/2357724X06112018336.

SOARES, J.M. et al.(2008) Instrumentação computacional e realimentação no processo de avaliação para o ensino de matemática: o conhecimento de função real como estudo de caso. Simpósio Brasileiro de Informática na Educação - SBIE, p. 360-369.

Wanderley, Y. (2017). Apropriações tecnológicas no ensino e aprendizagem: As experiências dos educadores da Rede Anísio Teixeira. Disponível em: http://repositorio.ufba.br/ri/handle/ri/22983.

Link para acessar as referências de todos os trabalhos lidos no MSL: https://docs.google.com/document/d/1z0qCLwkajW_thQU2Qn7IEx6WymFLMTjf1 A5rnR9WG9A/edit?usp=sharing. 